\title{
Modulation of Gut Microbiota Composition and Short-Chain Fatty Acid Synthesis by Mogroside V in an In Vitro Incubation System
}

\author{
Ruiming Xiao, Wanci Liao, Guangjuan Luo, Zhenning Qin, Shuangyan Han, and Ying Lin*
}

Cite This: ACS Omega 2021, 6, 25486-25496

Read Online

ABSTRACT: Mogroside V (MV), a sweetener, is one of the major components inSiraitia grosvenorii. In our research, after in vitro incubation with MV for $24 \mathrm{~h}$, the human gut microbiota diversity changed, with an enrichment of the genera Bacteroides, Lactobacillus, Prevotella, Megasphaera, and Olsenella and the inhibition of Clostridium XIVa, Dorea, and Desulfovibrio. Moreover, the synthesis of short-chain fatty acids, such as acetate, propionate, and butyrate, was increased by gut microbiota. According to ultraperformance liquid chromatography-mass spectrometry (UPLC-MS) analysis, MV was decomposed into secondary mogrosides, such as mogroside II/I and mogrol, by gut microbiota. Enhanced antioxidant abilities of the metabolites were found in the broth. The results suggested that $\mathrm{MV}$, as a potential prebiotic, could benefit human health through its interaction with gut microbiota.

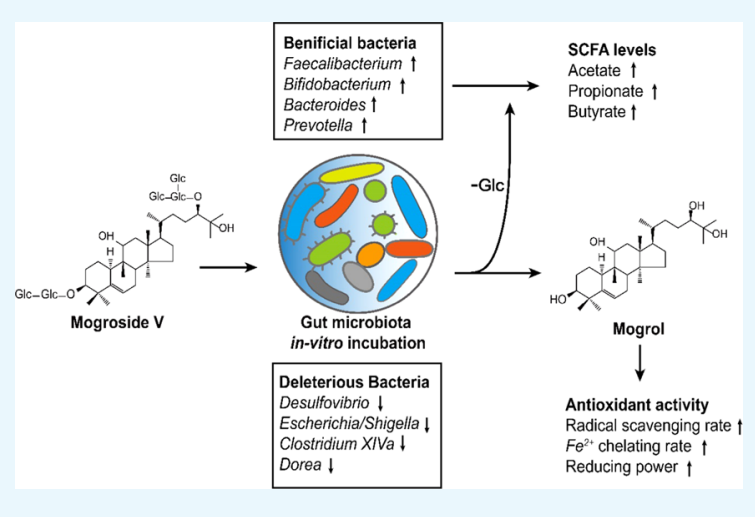

\section{INTRODUCTION}

Siraitia grosvenorii, called Luohanguo in southern China, is the fruit of lianas belonging to Cucurbitaceae and genus of Siraitia. The dried fruit is widely used for daily drinking and as traditional Chinese medicine. It contains various functional components, such as triterpenoids and flavonoids, among which the triterpene glycoside mogroside $\mathrm{V}(\mathrm{MV})$ is one of the major bioactive components, which is constructed by linking mogrol with five glucopyranosides. ${ }^{1}$ As an important sweetener source and food additive that is approximately 300 times sweeter than sucrose and low in calories, MV has been applied to food processes in China, Japan, and the United States. ${ }^{2,3}$ $\mathrm{MV}$ is considered to be a health-promoting functional component based on present in vitro research studies and animal experiments, it has been reported to decrease blood glucose level in HepG2 cells, ${ }^{4}$ lower blood lipid in diabetic mice, ${ }^{5}$ and exhibit antioxidative in vitro ${ }^{5,6}$ and inhibitory effect of skin tumors in peroxynitrite- and 12-O-tetradecanoylphorbol-13-acetate-induced mouse. ${ }^{6}$ Recently, purified mogrosides (e.g., MV) derived from monk fruit extracts were reported to be metabolized in vitro by the gut microbiota to form a common and terminal deglycosylated metabolite, mogrol. ${ }^{7}$ Nevertheless, the effects of the interactions between MV and gut microbiota on human health are still unclear.

To date, the prebiotic functions of polysaccharides derived from plants and fungi have received wide attention. ${ }^{8,9}$ These components cannot be metabolized by the host directly; as a result, the gut microbiota helps to digest the nutrients to improve their bioavailabilities and functions. ${ }^{10}$ Past studies have reported the differences in the secondary metabolites produced from $S$. grosvenorii extract by gut microbiota of healthy and diabetic donors. ${ }^{11}$ However, the effects of MV on gut microbiota have received little attention. In the past few years, active plant components, such as hesperetin, have been confirmed to modulate gut microbiota diversity and affect host health by releasing multifarious metabolites or short-chain fatty acids (SCFAs), ${ }^{12}$ which are a series of products from gut microbial fermentation and possible signaling molecules that regulate host immunoreactions. ${ }^{13}$ For instance, dietary fiber supplementation with fermentable prebiotics in diabetic patients altered multiple bacterial metabolites and enriched SCFA-producing bacteria, ${ }^{14}$ which are known as potential probiotics. Polysaccharides from Dendrobium officinale were reported to relieve dextran sulfate sodium-induced colitis, modulate SCFA levels, and contribute to microbiota abundance, such as Bacteroides, Lactobacillus, and Ruminococcaceae. ${ }^{15}$ Understandably, gut microbial diversity is closely connected with the metabolic diversity of xenobiotic compounds, since the microbial digestion of carbohydrates and their derivatives represents one of the most important forms of metabolism. ${ }^{16}$ There are thousands of glucoside hydrolases encoded in the gut microbial genome, especially in Bacteroides and Firmicutes, which account for $86 \%$ of the total amount of genes. ${ }^{17,18}$ These intestinal bacteria could help

Received: July 2, 2021

Accepted: August 5, 2021

Published: September 21, 2021 


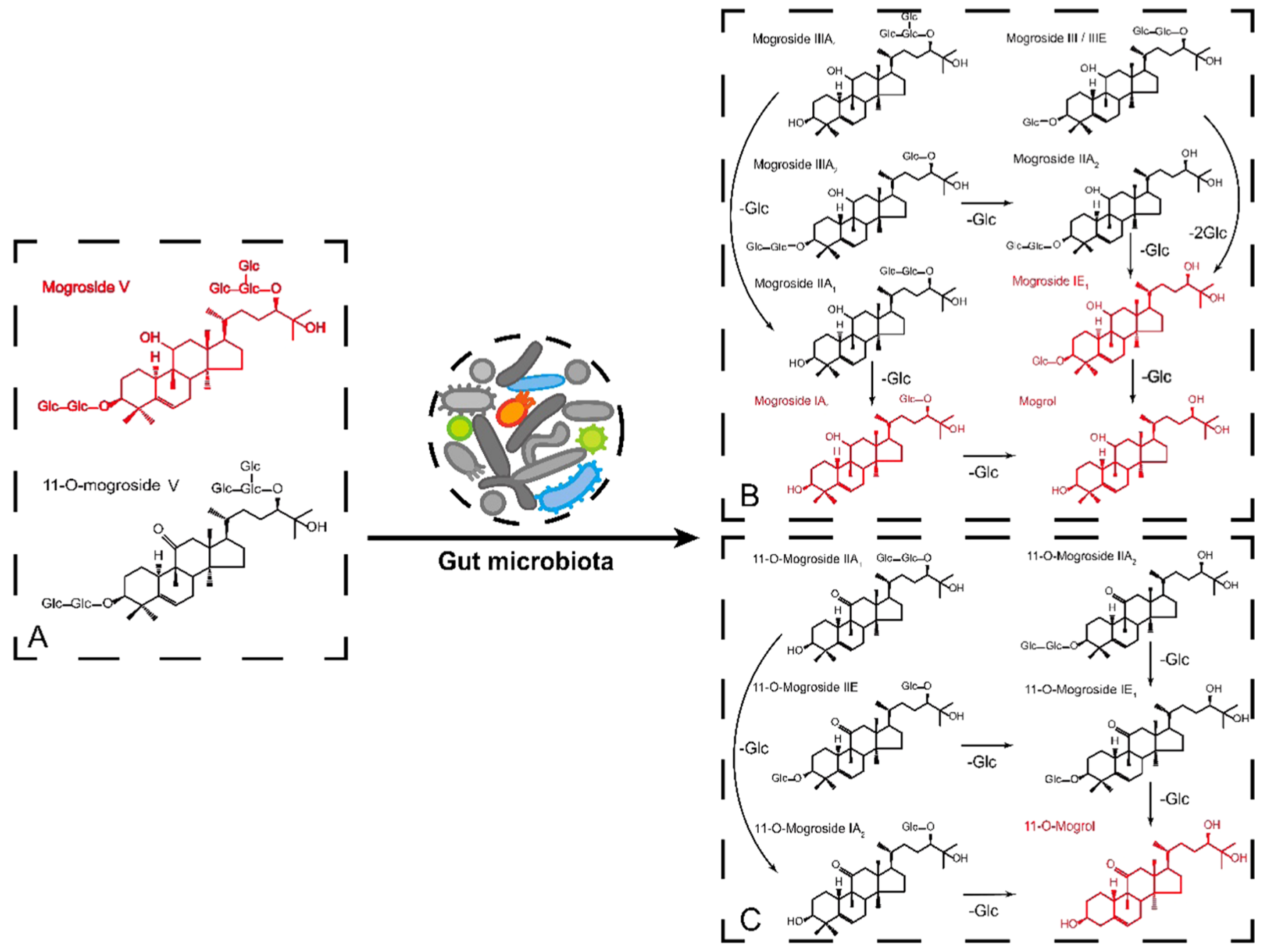

Figure 1. Transformation of MV and 11-O-mogroside V by the gut microbiota. (A) Components in $0 \mathrm{~h}$ incubation broth. (B) Metabolites of MV after $24 \mathrm{~h}$ of incubation. (C) Metabolites of 11-O-mogroside V after $24 \mathrm{~h}$ of incubation. Compounds marked in red reflect the major components in the broth.

hydrolyze these polysaccharides into monosaccharides, the latter being an available substrate for fermentation by intestinal bacteria to produce SCFAs.

In our research, $\mathrm{MV}$, a mogroside purified from $S$. grosvenorii, was digested by incubation with the gut microbiota in vitro to examine the effects of microbial composition with absolute copies of each bacterial taxon and SCFA production and formed metabolites that were detected by ultraperformance liquid chromatography-mass spectrometry (UPLC-MS) to investigate the transformational significance of MV. The results provided an understanding of the potential prebiotic functions of MV.

\section{RESULTS AND DISCUSSION}

Metabolism of MV by In Vitro Incubation with Gut Microbiota. MV purified from S. grosvenorii, in which the purity of the MV powder was $90.08 \pm 1.09 \%$, was incubated in vitro with gut microbiota for $24 \mathrm{~h}$. To observe the metabolism of MV, UPLC coupled with ultrahigh-resolution MS was used to detect the metabolites in the supernatant and sediment of the broth. As shown in Figure 1, MV was mainly identified in the broth before incubation. Then, the amount of MV dramatically decreased after incubation for $24 \mathrm{~h}$, while some secondary metabolites appeared in the sediment, such as mogroside III, mogroside II, mogroside I, and mogrol. 11-OMogroside V, which had a low content, has a similar metabolic pathway to produce 11-O-mogrosides II and I and 11-Omogrol. The MS response values of the other mogrosides are listed in Table S1. As a result, these metabolites gradually separated from the supernatant into the sediment of the broth over $24 \mathrm{~h}$ of incubation due to their reduced solubility. The results showed that deglycosylation of MV dominated the in vitro incubation by the gut microbiota. Interestingly, secondary mogrosides, such as mogroside IV, appeared in the broth during the first $6 \mathrm{~h}$, but its content decreased afterward (Figure S1D), indicating that the microbial deglycosylation was a stepby-step process. Oxidization and dehydrogenation reactions were not found to occur, which verified that these metabolites of MV were more common in healthy donors. ${ }^{11}$ Similarly, in recent research, MV cultured with intestinal bacteria also produced these metabolites, among which 11-oxo-mogrol was identified to protect cultured neurons against MK801 treatment in vitro; ${ }^{19}$ however, 11-oxo-mogrol was not a major metabolite of MV in our research according to Table S1.

Previous studies have also reported that triterpene glycosides such as ginsenoside Rb1 could be hydrolyzed to $20(S)$ protopanaxadiol and compound $\mathrm{K}$, which can be well absorbed by gut epithelial cells and play a role with higher functional 
Table 1. Alpha Diversity Parameters of Different Treatment Groups ${ }^{a}$

$\begin{array}{lcccc}\text { groups } & \text { Chao1 } & \text { ACE } & \text { Shannon } & \text { Simpson } \\ \text { Control } & 419.52 \pm 12.15 \mathrm{a} & 420.09 \pm 12.50 \mathrm{a} & 4.30 \pm 0.02 \mathrm{a} & (5.82 \pm 0.35) \times 10^{-2} \mathrm{a} \\ \text { FOS } & 235.93 \pm 34.67 \mathrm{~b} & 236.35 \pm 34.78 \mathrm{~b} & 3.56 \pm 0.04 \mathrm{c} & (6.05 \pm 0.13) \times 10^{-2} \mathrm{a} \\ \text { MV } & 259.62 \pm 10.20 \mathrm{~b} & 260.04 \pm 10.04 \mathrm{~b} & 3.74 \pm 0.01 \mathrm{~b} & (5.03 \pm 0.05) \times 10^{-2} \mathrm{~b}\end{array}$

${ }^{a}$ Different lowercase letters indicate significant differences $(P<0.05)$ among different groups.

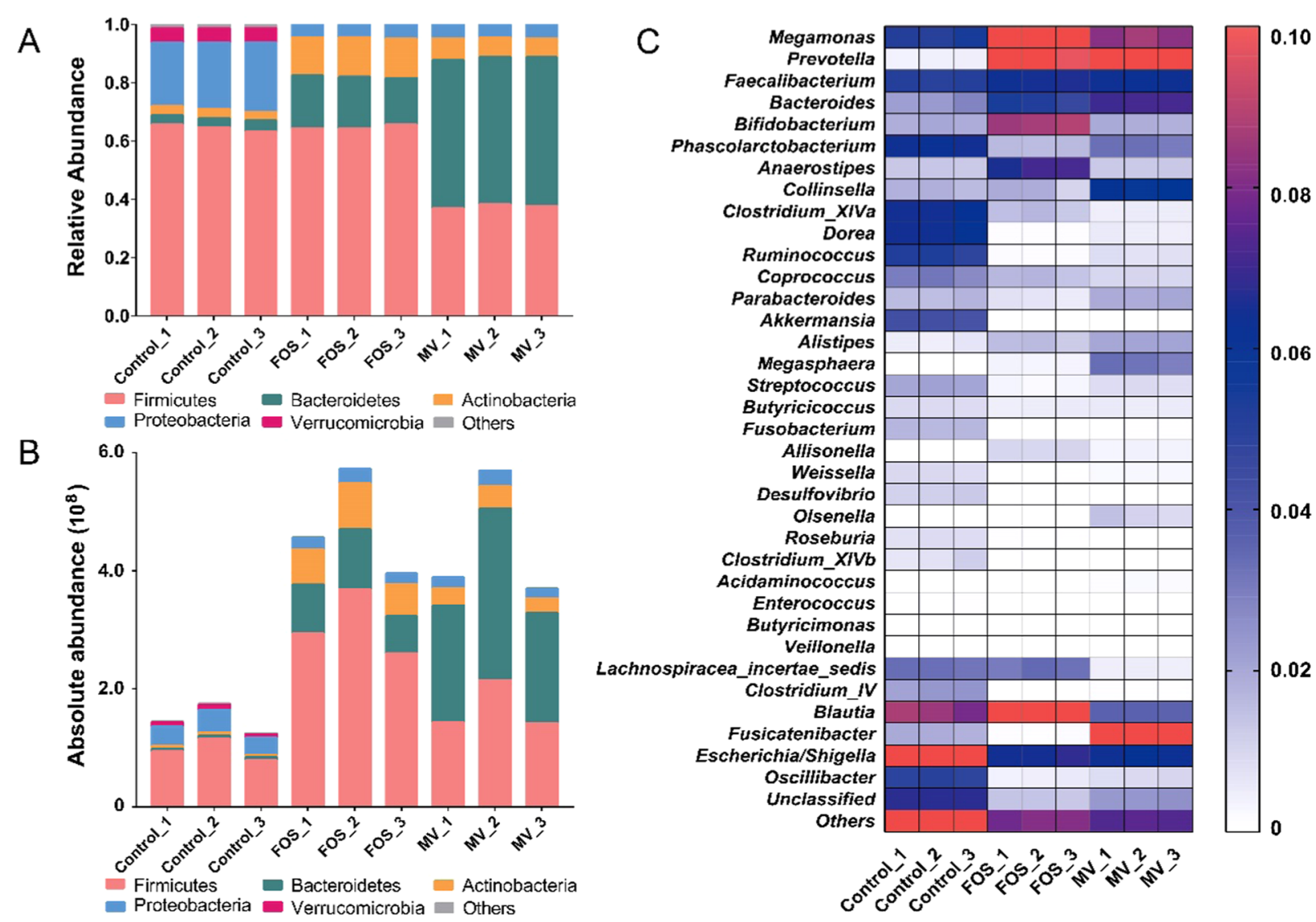

Figure 2. (A) Relative abundance of gut microbiota at the phylum level. (B) Absolute abundance (copy number) of gut microbiota at the phylum level. (C) Heat map of gut microbiota at the genus level. FOS.

efficiency. $^{20}$ Similar results were obtained in our study; metabolites containing mogroside III, II, and I showed available antihyperglycemic and antihyperlipidemic effects on T2DM rats. Moreover, mogrol, the most abundant component in the broth, helped alleviate colitis through the adenosine monophosphate-activated protein kinase (AMPK) and NF- $\kappa \mathrm{B}$ pathways, ${ }^{21}$ suggesting that MV might be digested by the gut microbiota to generate metabolites that benefit human health.

Additionally, simulated digestion in saliva, gastric juice, and small intestinal juice was conducted to examine the stability of MV. The results suggested that MV could stably pass through saliva and simulated gastric and small intestinal conditions (Figure S1A-C). Humans lack $\beta$-glucoside hydrolase, which is encoded in the gut microbiota genome; ${ }^{22}$ therefore, it was speculated that MV might be metabolized not by the human digestive enzymes in vivo but commensal bacteria in the gut tract. However, due to the lack of bioavailability information of $\mathrm{MV}$, the metabolic mechanism still needs to be verified by further pharmacokinetics research in vivo.

Modulation of Gut Microbiota Diversity by MV. The gut microbiota of the fecal slurry was anaerobically incubated in a basic nutrient growth medium (BNM) with $\mathrm{MV}$ and fructooligosaccharide (FOS), and a sample without any additive was set as a control. After $24 \mathrm{~h}$ of incubation, each sample was independently harvested to extract genomic DNA for $16 \mathrm{~S}$ rRNA sequencing of the V3-V4 region. The rarefaction curve and Shannon curve indicated that the sequencing depth covered nearly all of the microorganisms, and principal coordinates analysis ( $\mathrm{PCoA}$ ) analysis reflected the significant difference between all treatment groups (Figure S2). Optimized sequences were clustered according to the similarity of $97 \%$ and blasted with the Ribosomal Database Project (RDP) database to identify the taxa. The alpha diversity results are shown in Table 1 . The Chao1, ACE, and Shannon indexes in the MV and FOS groups were significantly lower than those of the control group, while the Simpson index was higher in the FOS group. It was therefore suggested that the advantageous bacteria were enriched by the addition of MV.

The relative differences in the gut microbiota after incubation with various media are presented in Figure 2A, and a bar plot was drawn from the data with microbes with an abundance greater than $1 \%$. At the phylum level, the predominant taxa were Firmicutes, Bacteroidetes, Proteobacteria, 


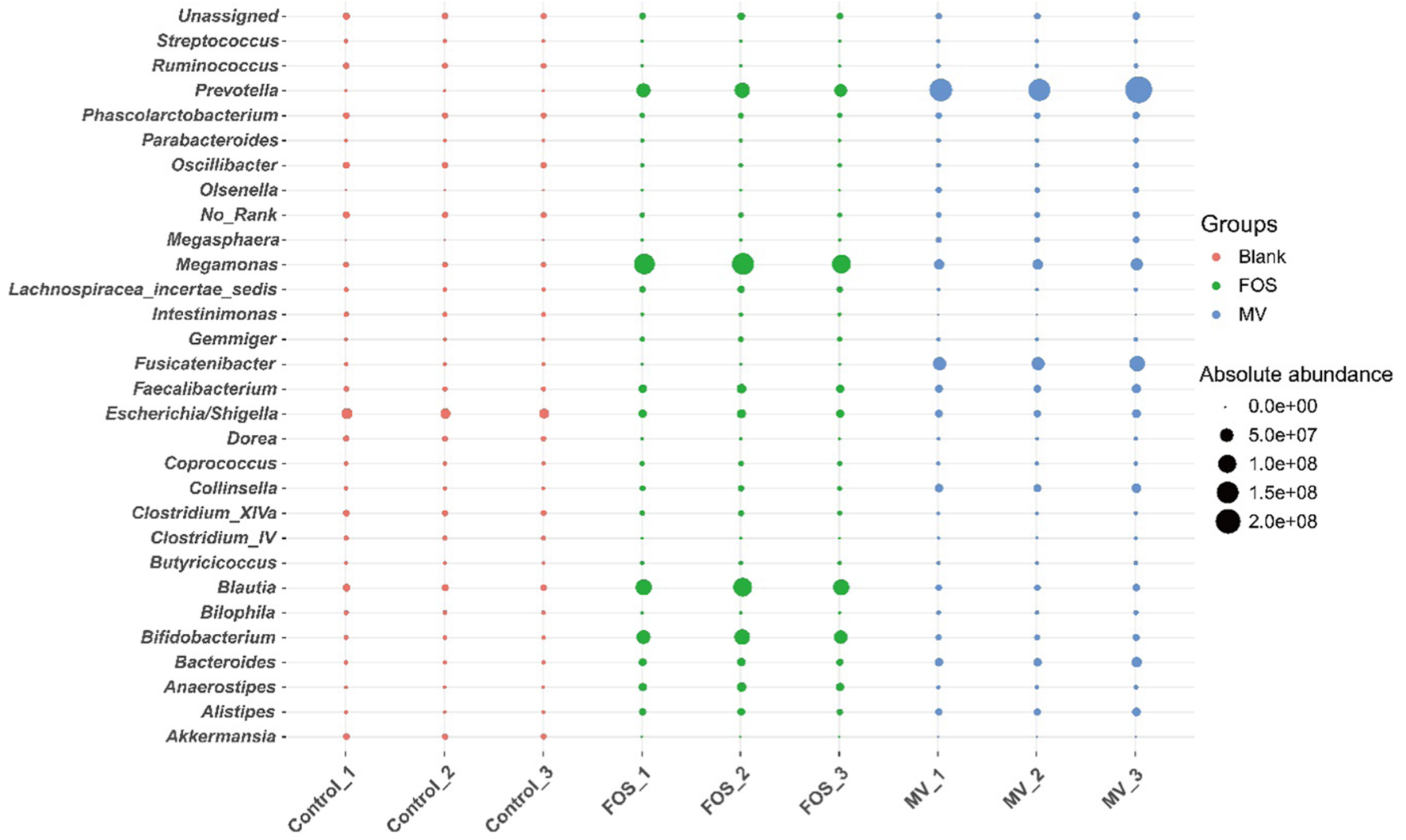

Figure 3. Differences of absolute abundance of gut microbiota at genus level between MV, FOS, and control.

and Actinomycetes. In particular, the relative abundances of Actinomycetes and Bacteroidetes increased after MV addition, whereas those of Proteobacteria and Firmicutes decreased in the MV and FOS groups. By comparing the relative and absolute abundance results, however, a discrepant conclusion could be drawn due to the differences in absolute abundance among the $\mathrm{MV}$, FOS, and control samples. For instance, as shown in Figure $2 \mathrm{~B}$, the absolute copies of total bacteria from both samples were significantly higher than that in the control, although the relative abundance of Firmicutes decreased in the sample with added MV. Therefore, the number of OTUs from Firmicutes increased in the sample with added MV, which presented the advantage of revealing actual microbial changes by absolute quantification of amplicon sequencing. Furthermore, we found that the addition of MV increased the abundance of Firmicutes, Bacteroidetes, and Actinomycetes, while FOS only increased the abundance of Actinomycetes and Bacteroidetes. On the other hand, Proteobacteria was notably reduced in the samples with added MV. Similar results were also investigated in the latest study, additive of monk fruit extract in yogurt increased the relative abundance of Bacteroidetes and reduced Firmicutes and regulated blood glucose in rats with $\mathrm{T}_{2} \mathrm{DM}{ }^{23}$ Recently, a large-scale population study of gut microbiota showed that the majority of Proteobacteria were positively correlated to the occurrence of metabolic syndrome while most Bacteroidetes were more prevalent in healthy individuals, ${ }^{24}$ indicating the potential health functions of MV through the modulation of the gut microbiota composition.

At the genus level (Figure 2C), the taxa were mainly Bacteroides, Megamonas, Bifidobacterium, Prevotella, Blautia, and so on. Clearly, compared with the control, the relative abundances of Prevotella, Megamonas, Bacteroides, Olsenella, and Faecalibacterium were enriched in the MV-added sample. There were a few differences in the added FOS sample, in which Bifidobacterium, Anaerostipes, and Blautia were specifically enriched.

To illustrate the absolute copy differences in specific bacteria among samples, Figure 3 lists the top 30 significantly changed genera. The MV group obviously contributed to 12 taxa, mainly Megamonas, Faecalibacterium, Bacteroides, Megasphaera, Prevotella, Olsenella, and others. The integrated enriched bacteria were as follows: Bacteroides (10.31-fold), Lactobacillus (19.47-fold), Faecalibacterium (3.81-fold), Prevotella (421.90fold), Megamonas (6.29-fold), Olsenella (243.76-fold), Megasphaera (3693.62-fold), Collinsella (10.07-fold), Fusicatenibacter (36.33-fold), Parabacteroides (3.51-fold), Alistipes (11.57-fold), and Allisonella (2564.42-fold). The bacteria whose relative abundances dramatically decreased were Clostridium XIVa, Dorea, Shigella, Desulfovibrio, and Fusobacterium. As reported by a previous survey, ${ }^{25}$ FOS could obviously enrich bacteria, including Prevotella and Bacteroides, as was observed in our study, which helped to hydrolyze and utilize the indigestible polysaccharides and simultaneously promote the growth of probiotics such as Lactobacillus and Bifidobacterium in return. Similar results were also found in our study. MV significantly improved the amount of Faecalibacterium, Prevotella, and Bacteroides and slightly increased the abundance of Lactobacillus, which were identified to contain nextgeneration probiotic species and are positively connected with gut health. ${ }^{26}$ Fucatenibacer was also found to be highly enriched in the MV group, but few studies have been done on this genus. 
Table 2. Concentrations of SCFAs in Incubation Solutions at Different Time Points ${ }^{a}$

\begin{tabular}{|c|c|c|c|c|c|c|}
\hline \multirow[b]{2}{*}{ SCFAs (mM) } & \multirow[b]{2}{*}{ samples } & \multicolumn{5}{|c|}{ anaerobic incubation time $(\mathrm{h})$} \\
\hline & & 0 & 3 & 6 & 12 & 24 \\
\hline \multirow[t]{3}{*}{ acetate } & control & $0.31 \pm 0.02$ & $1.02 \pm 0.04 \mathrm{a}$ & $2.14 \pm 0.08 \mathrm{a}$ & $2.89 \pm 0.10 \mathrm{a}$ & $4.16 \pm 1.19 \mathrm{a}$ \\
\hline & FOS & & $1.89 \pm 0.04 \mathrm{~b}$ & $4.22 \pm 0.20 \mathrm{~b}$ & $7.78 \pm 0.29 b$ & $9.47 \pm 0.34 b$ \\
\hline & MV & & $2.18 \pm 0.50 \mathrm{~b}$ & $6.30 \pm 0.28 c$ & $11.66 \pm 0.56 \mathrm{c}$ & $15.34 \pm 0.68 \mathrm{c}$ \\
\hline \multirow[t]{3}{*}{ propionate } & control & $0.29 \pm 0.02$ & $0.84 \pm 0.01 \mathrm{a}$ & $1.61 \pm 0.03 \mathrm{a}$ & $2.98 \pm 0.11 \mathrm{a}$ & $2.68 \pm 0.53 \mathrm{a}$ \\
\hline & FOS & & $3.78 \pm 0.09 \mathrm{c}$ & $11.11 \pm 0.29 \mathrm{c}$ & $10.18 \pm 0.33 \mathrm{c}$ & $10.53 \pm 0.79 c$ \\
\hline & MV & & $1.46 \pm 0.08 \mathrm{~b}$ & $3.73 \pm 0.10 b$ & $6.41 \pm 0.22 b$ & $8.43 \pm 0.28 b$ \\
\hline \multirow[t]{3}{*}{ butyrate } & control & $0.33 \pm 0.01$ & $1.01 \pm 0.01 \mathrm{a}$ & $1.82 \pm 0.02 \mathrm{a}$ & $2.51 \pm 0.05 \mathrm{a}$ & $2.33 \pm 0.06 \mathrm{a}$ \\
\hline & FOS & & $1.56 \pm 0.01 \mathrm{~b}$ & $4.49 \pm 0.07 \mathrm{c}$ & $4.94 \pm 0.08 b$ & $3.35 \pm 0.12 b$ \\
\hline & MV & & $1.53 \pm 0.19 b$ & $3.42 \pm 0.06 b$ & $5.63 \pm 0.13 c$ & $6.98 \pm 0.13 c$ \\
\hline \multirow[t]{3}{*}{ isobutyrate } & control & M & nd & $0.04 \pm 0.01 \mathrm{a}$ & $0.95 \pm 0.02 b$ & $1.01 \pm 0.02 \mathrm{~b}$ \\
\hline & FOS & & nd & nd & nd & nd \\
\hline & MV & & nd & $0.08 \pm 0.01 b$ & $0.16 \pm 0.01 \mathrm{a}$ & $0.15 \pm 0.01 \mathrm{a}$ \\
\hline \multirow[t]{3}{*}{ valerate } & control & nd & nd & $0.10 \pm 0.01 \mathrm{a}$ & $0.39 \pm 0.05 c$ & $0.22 \pm 0.03 b$ \\
\hline & FOS & & $0.05 \pm 0.01 \mathrm{a}$ & $0.09 \pm 0.01 \mathrm{a}$ & $0.10 \pm 0.01 \mathrm{a}$ & $0.06 \pm 0.01 \mathrm{a}$ \\
\hline & MV & & nd & $0.11 \pm 0.01 \mathrm{a}$ & $0.22 \pm 0.01 b$ & $0.31 \pm 0.01 c$ \\
\hline \multirow[t]{3}{*}{ isovalerate } & control & nd & nd & $0.29 \pm 0.01 b$ & $2.17 \pm 0.03 c$ & $2.24 \pm 0.01 c$ \\
\hline & FOS & & nd & $0.05 \pm 0.01 \mathrm{a}$ & $0.06 \pm 0.01 \mathrm{a}$ & $0.08 \pm 0.05 a$ \\
\hline & MV & & nd & $0.30 \pm 0.01 b$ & $0.53 \pm 0.01 \mathrm{~b}$ & $0.58 \pm 0.01 b$ \\
\hline
\end{tabular}

${ }^{a}$ Different lowercase letters indicate significances $(P<0.05)$ among groups; nd, not detected.

The high enrichment of $B$. uniformis from Bacteroides might be related to the hydrolysis of MV due to the $\beta$-glycosidase encoded in its bacterial genome. ${ }^{27}$ Another enriched species, $B$. vulgatus, was reported to reduce the production of lipopolysaccharide in the gut and suppress proinflammatory immune responses. $^{28}$ In addition, supplementation with Faecalibacterium prausnitzii was confirmed to improve liver health and reduce inflammation in mice fed a high-fat diet. ${ }^{29}$ Although other enriched genera was reported to be connected with the fermentation of carbohydrates and known as SCFAproducing bacteria, such as Megasphaera and Megamonas, ${ }^{30,31}$ their probiotic function still remains to be confirmed. As for the reducing taxa, some species from Clostridium XIVa have been considered to contain opportunistic pathogens and are inhibited by fucosylated chondroitin sulfate. ${ }^{32}$ Dorea and Desulfovibrio are considered proinflammatory genera, ${ }^{3,34}$ and the latter is a producer of $\mathrm{H}_{2} \mathrm{~S}$, which regulates inflammatory processes. Overall, the above results indicate that MV has potential prebiotic functions to improve health through interactions with the gut microbiota.

Enhancement of Short-Chain Fatty Acid Synthesis in the Gut Microbiota after the Addition of MV. As reported, bacteria from Firmicutes and Bacteroidetes contain the most glucoside hydrolases, which hydrolyze typical glucosides, such as xylan and arabinoxylan, and produce metabolites to enhance the growth of Bifidobacterium. ${ }^{35}$ This mechanism might explain the coenrichment of Bacteroides, Bifidobacterium, and other genera. In addition, hydrolyzed glucosides act as an available substrate for bacteria, such as Faecalibacterium, Bacteroides, and Megasphaera, to produce SCFAs during anaerobic fermentation, mainly acetate, propionate, and butyrate.

To investigate the correlation between the metabolism of glucosides and the production of SCFAs in the gut microbiota, the contents of acetate, propionate, butyrate, isobutyrate, valerate, and isovalerate were determined in the abovementioned three groups. As presented in Table 2, the contents of SCFAs significantly increased in the MV and FOS samples during $24 \mathrm{~h}$ of incubation. However, a difference in SCFAs was observed between the MV and FOS samples. The increase in acetate in the sample with added MV was significantly higher than that in the FOS sample after $24 \mathrm{~h}$ of incubation. The concentration of acetate in the FOS sample increased to 9.47 $\pm 0.34 \mathrm{mM}$, while that in MV increased to $15.34 \pm 0.68 \mathrm{mM}$. For propionate, the FOS-added sample displayed an increase to $10.53 \pm 0.79 \mathrm{mM}$, showing a significant difference from the sample with added MV. Moreover, butyrate in the MV sample obviously increased compared with the FOS sample during the last $12 \mathrm{~h}$ of incubation, reaching $6.98 \pm 0.13 \mathrm{mM}$. In the latest research, a high dose of monk fruit extract in yogurt reinstated the concentration of acetate, propionate, and butyrate in T2DM rats, reflecting the potential correlation between mogrosides and host diseases since SCFAs were considered as health-related signaling molecules. For instance, acetate and butyrate possibly regulate the immune system through Gprotein-coupled receptors 43 and 109A and modulate insulin sensitivity through fatty acid receptors FFAR2 and FFAR3. ${ }^{36,37}$ In addition, butyrate can be utilized as an energy source by epithelial cells. ${ }^{38}$ Propionate was thought to reduce lipogenesis and cholesterol levels. ${ }^{39}$ Other SCFAs, such as isobutyrate, valerate, and isovalerate, were inhibited in the FOS group but improved in the MV group after $24 \mathrm{~h}$ of incubation, suggesting that MV significantly promoted the yield of branched-chain fatty acids (BCFAs), a series of protein fermentation products produced by the gut microbiota. SCFAs in the intestinal tract also contributed to inhibiting the growth of pathogenic bacteria; for example, adding valerate could restrain the development of Clostridioides difficile. ${ }^{40}$ Nevertheless, the healthy function of BCFAs remains unknown. Consequently, MV contributed to the number of SCFA-producing bacteria and supplied an available substrate for microbial fermentation. The ability to increase SCFA levels provided a new understanding of MV to improve gut health.

Enhancement of MV Antioxidation after In Vitro Incubation with Gut Microbiota. Free radicals are a general product of biochemical reactions in the body. However, superfluous radicals are likely to induce tissue damage or the development of host diseases. ${ }^{41}$ Plant active components, such 

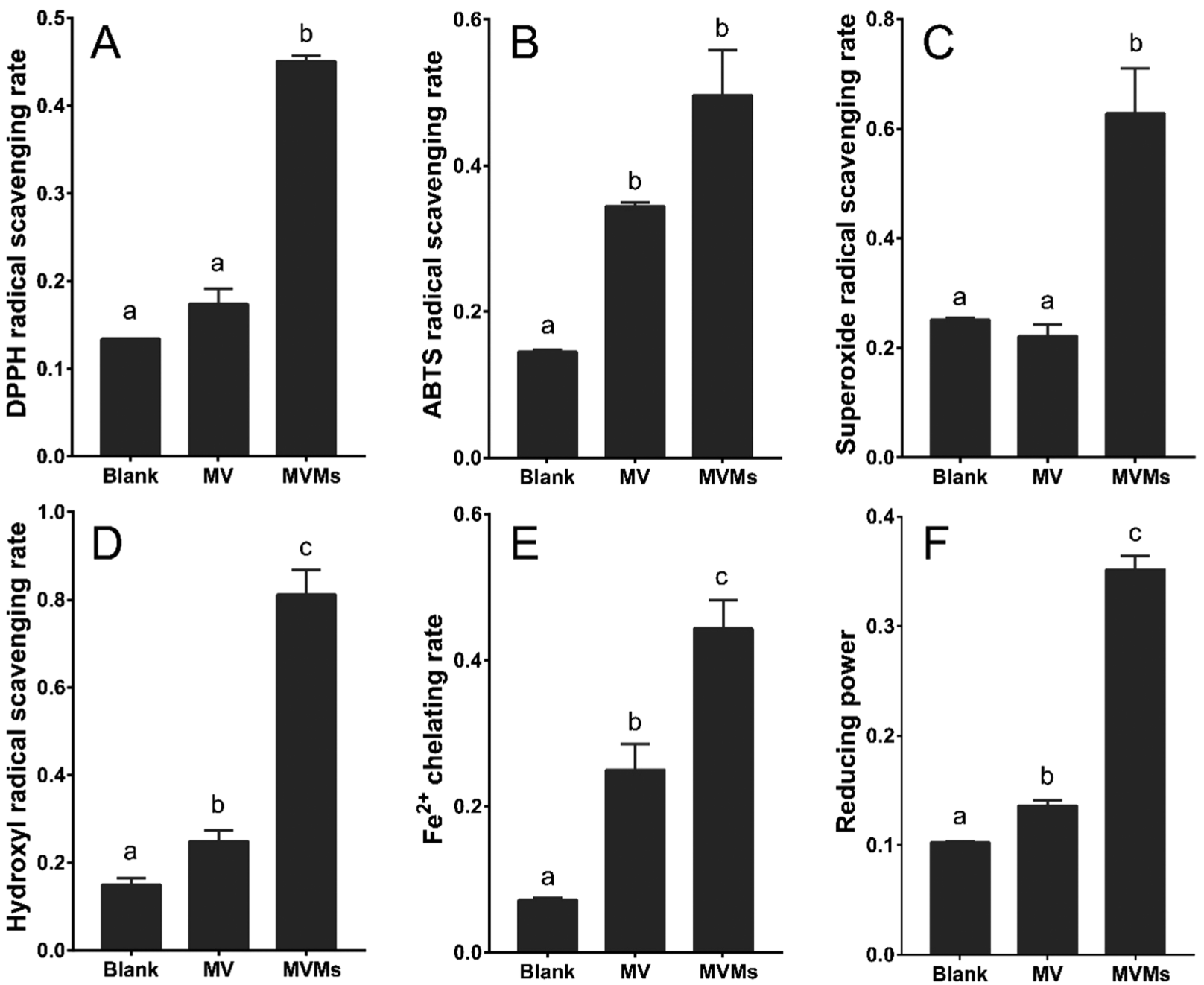

Figure 4. Differences between the antioxidant activities of MV and MVMs in (A) DPPH radical scavenging rate, (B) ABTS radical scavenging rate, (C) superoxide radical scavenging rate, (D) hydroxyl radical scavenging rate, $(\mathrm{E}) \mathrm{Fe}^{2+}$ chelating rate, and (F) reducing power.

as ginsenosides, have been verified to protect the host from an oxidative stress injury. ${ }^{42}$ Similarly, triterpenes have been reported to have potential antioxidant activity. ${ }^{43}$ Here, the antioxidant activity of MV and its metabolites mogroside $\mathrm{V}$ metabolite (MVMs) after in vitro incubation with gut microbiota were examined by 1,1-diphenyl-2-picrylhydrazyl (DPPH) radical scavenging activity, hydroxyl radical scavenging activity, superoxide radical scavenging activity, [2,2azinobis-(3-ethyl-benzothiazoline-6-sulfonic acid)] diammonium salt (ABTS) radical scavenging activity, $\mathrm{Fe}^{2+}$ chelating activity, and total reducing power.

After in vitro incubation of the gut microbiota for $24 \mathrm{~h}$, there was a dramatic rise in metabolites, as described in Table S1, and the antioxidant index, as presented in Figure 4A-F. The $\mathrm{DPPH}$ radical scavenging rate increased from $17.38 \pm 1.75$ to $45.11 \pm 0.57 \%(P<0.05)$, the hydroxyl radical scavenging rate increased from $24.88 \pm 2.52$ to $81.18 \pm 5.60 \%(P<0.05)$, the superoxide radical scavenging rate increased from $22.12 \pm 2.14$ to $62.82 \pm 8.18 \%(P<0.05)$, the ABTS radical scavenging rate increased from $34.42 \pm 0.53$ to $49.66 \pm 6.11 \%(P<0.05)$, the $\mathrm{Fe}^{2+}$ chelating rate increased from $25.01 \pm 3.58$ to $44.35 \pm$ $3.85 \%(P<0.05)$, and the total reducing power improved from $13.62 \pm 0.52$ to $35.16 \pm 1.26 \%(P<0.05)$. In recent decades, free radicals have been considered to be able to react with biological macromolecules such as DNA, membrane lipids, and proteins, causing oxidative modifications and loss of function. ${ }^{44}$ For MVMs, the scavenging rate of hydroxyl radicals, known as one of the most reactive free radicals, was nearly 3.3-fold higher than that of MV. Scavenging of DPPH, ABTS, and superoxide radicals also improved by 2.6-, 1.4-, and 2.8-fold, respectively, suggesting a promotion in the radical scavenging functions of MVMs. $\mathrm{Fe}^{2+}$ was active in the oxidation bioreaction and connected with radicals produced through the Fenton reaction. As presented in Figure 4E,F, the MVMs also showed stronger ferrous ion chelating activity and total reducing power than MV. Furthermore, $0.02 \mathrm{M}$ mogrol and MV were compared to confirm the increased antioxidant activity of major metabolites. The data from Figure S3A-F suggest that the scavenging of DPPH, ABTS, superoxide, and hydroxyl radicals by mogrol are 1.3-, 1.3-, 6.0-, and 1.4-fold than that by $\mathrm{MV}$, respectively, the $\mathrm{Fe}^{2+}$ chelating rate and the reducing power of mogrol were 1.8- and 1.4-fold than that of $\mathrm{MV}$, respectively, indicating that the enhanced antioxidant activity was related to the improved concentration of mogrol after $24 \mathrm{~h}$ of microbial incubation. However, the improved antioxidant activity seems not completely resulted from the production of mogrol. For instance, the DPPH and hydroxy radical scavenging rates of MVMs were higher than that of mogrol. A similar result was also found for the reducing power. These differences might be caused by the multiple antioxidant 
components in MVMs such as mogroside I and 11-O-mogrol, since the antioxidant activity was related to the position and numbers of hydroxy, steric effects, and molecular properties. ${ }^{45}$ The above results suggested that MVMs likely possess stronger bioactivities to protect gut homeostasis after degradation by the gut microbiota, for example, MV and 11-oxo-mogrol (one of the MV metabolites) prevented from the neuronal damages induced by dizocilpine maleate (MK801); ${ }^{19}$ nevertheless, the in vivo therapeutic effect of MV and its metabolites should be further revealed. Structurally, because most metabolites resulted from the deglycosylation of MV with the action of $\beta$-glucosaccharase encoded in the gut microbiota genome, it was possible that the increase in antioxidant ability might be connected to the positions of the hydroxy groups and their bond dissociation enthalpy. ${ }^{46}$ In summary, the production of stronger antioxidative metabolites from gut microbiota was investigated from a novel perspective to illustrate the healthy functions of MV.

\section{CONCLUSIONS}

MV could be metabolized by gut microbiota into secondary mogrosides that have stronger antioxidant abilities for gut health but cannot be digested by saliva, simulated gastric juice, or small intestinal juice. During gut microbial fermentation, the glucosides released from the deglycosylation of MV promoted the growth of Bifidobacterium, Bacteroides, Prevotella, Lactobacillus, Mitsuokella, Selenomonas, Megasphaera, and so on and reduced the potential pathogenic genera Dorea and Clostridium XIVa. Additionally, these glucosides serve as substrates to produce SCFAs, especially acetate, propionate, and butyrate. It was speculated that MV could safely reach the large intestine in vivo and modulate the gut microecosystem to produce SCFAs. MV has potential benefits to human health through interactions with gut microbiota and shows promise for development as a prebiotic food.

\section{MATERIALS AND METHODS}

Materials and Chemicals. The MV in this study was provided by Guilin Sanling Biological Technology Co., Ltd. (Guangxi Province, China) and was extracted and purified from S. grosvenorii (the purity of MV was further identified in the next section). The MV standard was purchased from DeSiTe Biological Technology Co. Ltd. (Chengdu, China). Fructooligosaccharide was purchased from Qiyun Biotechnology Co., Ltd. (Guangzhou, China). Pepsin and trypsin were purchased from Sigma Chemical Co., Ltd. (St. Louis, MO). Pancreatin and lipase were obtained from Yuanye Biotechnology Co., Ltd. (Shanghai, China). [2,2-Azinobis-(3-ethylbenzothiazoline-6-sulfonic acid)] diammonium salt (ABTS), 1,1-diphenyl-2-picrylhydrazyl (DPPH), phenazine methosulfate (PMS), nicotinamide adenine dinucleotide (NADH), nitroblue tetrazolium (NBT), and salicylic acid were purchased from Newprobe Biotechnology Co., Ltd. (Beijing, China). Standards of SCFAs (acetic, propionic, butyric, isobutyric, valeric, and isovaleric acids) and all other chemicals were obtained from Aladdin Industrial Inc. (Shanghai, China).

MV Quantification. A Waters high-performance liquid chromatography (HPLC) system (Waters, Milford, MA) was used to quantitatively detect MV. A Shimadzu Inertsil ODS-3 C18 column $\left(4.6 \times 250 \mathrm{~mm}^{2}, 5 \mu \mathrm{m}\right)$ and an ultraviolet detector were used in this study. Other parameters were as follows: The column oven was $35^{\circ} \mathrm{C}$, the mobile phases were water with $0.1 \%$ phosphoric acid (A) and acetonitrile (B), and the flow rate was $1 \mathrm{~mL} / \mathrm{min}$. The linear gradient conditions were $15-40 \% \mathrm{~B}(0-30 \mathrm{~min})$ and $40 \% \mathrm{~B}(30-40 \mathrm{~min})$. The injection volume was $10 \mu \mathrm{L}$. The detector wavelength was set to $210 \mathrm{~nm}$.

Simulated Saliva Digestion. Simulated saliva digestion followed the method reported in a previous study with a slight adjustment. ${ }^{47}$ Fresh saliva was collected from four healthy participants who did not take any antibiotics in the 3 months prior. They were required to rinse their mouths and discard the first minute of saliva. After collection, the saliva was centrifuged at $5000 \mathrm{rpm}$ for $10 \mathrm{~min}$ to collect the supernatant. An MV solution was prepared at $10 \mathrm{mg} / \mathrm{mL}$. Three groups were set for the experiment: (1) $4 \mathrm{~mL}$ of saliva was mixed with $4 \mathrm{~mL}$ of $\mathrm{MV}$; (2) $4 \mathrm{~mL}$ of saliva was mixed with $4 \mathrm{~mL}$ of deionized water; and (3) $4 \mathrm{~mL}$ of MV was mixed with $4 \mathrm{~mL}$ of deionized water. All reaction tubes were kept in a water bath at $37^{\circ} \mathrm{C}$. After $0,0.5,1$, and $2 \mathrm{~h}$, the samples were collected to measure the consumption of MV by HPLC.

Simulated Gastric Digestion. The simulated gastric digestion method was assembled according to a previous study with a slight adjustment. ${ }^{48}$ The gastric electrolyte solution (GES; $200 \mathrm{~mL}$ ) contained $0.62 \mathrm{~g}$ of $\mathrm{NaCl}, 0.22 \mathrm{~g}$ of $\mathrm{KCl}, 0.05 \mathrm{~g}$ of $\mathrm{CaCl}_{2}$, and $0.12 \mathrm{~g}$ of $\mathrm{NaHCO}_{3}$. Then, $1.0 \mathrm{~mL}$ of $\mathrm{CH}_{3} \mathrm{COONa}(1.0 \mathrm{~mol} / \mathrm{L}, \mathrm{pH} 5), 23.6 \mathrm{mg}$ of gastric pepsin, and $25 \mathrm{mg}$ of gastric lipase were added to $100 \mathrm{~mL}$ of GES, and the $\mathrm{pH}$ of the mixture was adjusted to 3.0 with a $0.1 \mathrm{M} \mathrm{HCl}$ solution to obtain the gastric juice. Three groups were set for the experiment: (1) $8 \mathrm{~mL}$ of gastric juice was mixed with $8 \mathrm{~mL}$ of $\mathrm{MV}$; (2) $8 \mathrm{~mL}$ of gastric juice was mixed with $8 \mathrm{~mL}$ of deionized water; and (3) $8 \mathrm{~mL}$ of MV was mixed with $8 \mathrm{~mL}$ of deionized water. The reaction tubes were kept in a water bath at $37{ }^{\circ} \mathrm{C}$. After $0,0.5,1,2,4$, and $6 \mathrm{~h}$, the samples were collected to measure the consumption of MV by HPLC.

Simulated Small Intestinal Digestion. The simulated small intestinal digestion method was conducted according to a previous study with a slight adjustment. ${ }^{47}$ The intestinal electrolyte solution (IES; $100 \mathrm{~mL}$ ) contained $0.54 \mathrm{~g}$ of $\mathrm{NaCl}$, $0.065 \mathrm{~g}$ of $\mathrm{KCl}$, and $0.033 \mathrm{~g}$ of $\mathrm{CaCl}_{2}$, and the $\mathrm{pH}$ of the IES was adjusted to 7.0 with a $0.1 \mathrm{M} \mathrm{NaOH}$ solution. Then, 200 $\mathrm{mL}$ of bile salt solution $(4 \%, \mathrm{w} / \mathrm{v}), 50 \mathrm{~mL}$ of pancreatin solution $(7 \%, \mathrm{w} / \mathrm{v})$, and $6.5 \mathrm{mg}$ of trypsin were mixed with 50 $\mathrm{mL}$ of IES, and the mixture was centrifuged at $8000 \mathrm{rpm}$ for 10 min. The supernatant was collected, and the $\mathrm{pH}$ was adjusted to 7.5 with a $0.1 \mathrm{M} \mathrm{NaOH}$ solution to obtain the intestinal juice. The reaction systems were set at a ratio of 10:3 (v/v), and the mixture composition, sampling, and detection of $\mathrm{MV}$ were carried out as described in section 2.3.

In Vitro MV Incubation with the Gut Microbiota. Fecal sample collection methods were approved by the ethics committee of the First Affiliated Hospital of Guangzhou University of Chinese Medicine (Guangzhou, China). Fecal samples were obtained from four healthy participants (two males and two females) aged 20-26 years, and none of them had a history of antibiotic use within the 3 months prior to this research. The fecal samples were premixed and evenly resuspended in 10 volumes $(\mathrm{w} / \mathrm{v})$ phosphate-buffered saline (PBS) (0.1 M, pH 7.0) to obtain a mixed fecal slurry. The in vitro incubation of $\mathrm{MV}$ by the gut microbiota followed the method reported in a previous study, with a slight adjustment. ${ }^{32}$ In brief, the microbial community incubation was based on a basic nutrient growth medium (BNM): $2.0 \mathrm{~g} / \mathrm{L}$ yeast extract, $2.0 \mathrm{~g} / \mathrm{L}$ peptone, $0.1 \mathrm{~g} / \mathrm{L} \mathrm{NaCl}, 0.04 \mathrm{~g} / \mathrm{L}$ 
$\mathrm{KH}_{2} \mathrm{PO}_{4}, 0.04 \mathrm{~g} / \mathrm{L} \mathrm{K}_{2} \mathrm{HPO}_{4}, 0.01 \mathrm{~g} / \mathrm{L} \mathrm{MgSO} \mathrm{H}_{4} \cdot 7 \mathrm{H}_{2} \mathrm{O}, 0.01 \mathrm{~g} / \mathrm{L}$ $\mathrm{CaCl}_{2}, 2 \mathrm{~g} / \mathrm{L} \mathrm{NaHCO}{ }_{3}, 0.02 \mathrm{~g} / \mathrm{L}$ hemin, $0.5 \mathrm{~g} / \mathrm{L}$ cysteine- $\mathrm{HCl}$, $0.5 \mathrm{~g} / \mathrm{L}$ bile salts, $2.0 \mathrm{~mL} / \mathrm{L}$ Tween $80,1.0 \mathrm{~mL} / \mathrm{L} 1 \%$ resazurin solution, and $10 \mu \mathrm{L} / \mathrm{L}$ vitamin $\mathrm{K}$ solution. Then, $\mathrm{MV}$ and fructooligosaccharide (FOS; positive control) were added to the BNM medium at final concentrations of $10 \mathrm{mg} / \mathrm{mL}$, and the group with neither MV nor FOS was set as the control. One milliliter of the mixed fecal slurry was inoculated in an airtight serum bottle with $25 \mathrm{~mL}$ of BNM broth each group was inoculated in triplicate as parallel experiments. Incubation was carried out under anaerobic conditions at $37^{\circ} \mathrm{C}$, and at 0 , $3,6,12$, and $24 \mathrm{~h}$, the samples were collected for further study. Each experiment was performed in triplicate.

UPLC-MS Detection of MV Metabolites. Mass spectrometry analysis was carried out with a high-resolution $Q$ Exactive Plus Orbitrap MS system connected to a Dionex Ultimate 3000 UHPLC system (Thermo Fisher Scientific, Waltham, MA). MV and its metabolites were separated on the UPLC system equipped with a Hypersil GOLD C18 column $\left(100 \times 2.1 \mathrm{~mm}^{2}, 1.9 \mu \mathrm{m}\right.$; Thermo Fisher Scientific, Waltham, MA). The mobile phase was $0.1 \%$ formic acid (v/v, solvent A) and methanol (solvent B) with an injection volume of $5 \mu \mathrm{L}$, and the flow rate was $0.35 \mathrm{~mL} / \mathrm{min}$. The gradient process was as follows: $0-1 \mathrm{~min}, 2 \%$ solvent $B$ (held for $1 \mathrm{~min}$ ); $1-9 \mathrm{~min}$, from 2 to $98 \%$ solvent B; and 9-12 min, $98 \%$ solvent B (held for $3 \mathrm{~min})$. The column oven was set to $35{ }^{\circ} \mathrm{C}$. The electrospray ionization (ESI) parameters were set as follows: spray voltage, $3 \mathrm{kV}$; capillary temperature, $320{ }^{\circ} \mathrm{C}$; sheath gas flow rate, $40 \mathrm{~L} / \mathrm{h}$; aux gas flow rate, $10 \mathrm{~L} / \mathrm{h}$; and scan range, $\mathrm{m} /$ $z 133.4$ to 2000 . Ionization was conducted in the negative-ion mode. All data obtained were analyzed by Compound Discovery software (Thermo Fisher Scientific, Waltham, $\mathrm{MA}$ ), and the results were compared with those of a previous data set. $^{49}$

Detection of Antioxidant Activities of MV Metabolites In Vitro. Detection of Radical Scavenging Activity. The scavenging rates of $\mathrm{DPPH}, \mathrm{ABTS}^{+}$, hydroxyl, and superoxide radicals were measured according to the previous research. ${ }^{50}$ $\mathrm{MV}$ and its $24 \mathrm{~h}$ metabolites were dissolved in methanol to afford $10 \mathrm{mg} / \mathrm{mL}$ solutions. The antioxidant activities are expressed as the percent scavenging rate calculated by the following formula

$$
\text { radical scavenging rate }(\%)=\left(1-\frac{\mathrm{abs}_{1}-\mathrm{abs}_{2}}{\mathrm{abs}_{0}}\right) \times 100
$$

where $a b s_{0}$ is the abs of the control, $a b s_{1}$ is the abs of the samples, and $\mathrm{abs}_{2}$ is the abs under the same conditions as those of $\mathrm{abs}_{1}$ after replacing the radical solution with the control.

Detection of $\mathrm{Fe}^{2+}$ Chelating Activity. The $\mathrm{Fe}^{2+}$ chelating rate was measured by a reported assay with a slight adjustment. $^{50}$ Briefly, $50 \mu \mathrm{L}$ of the sample, $2.5 \mu \mathrm{L}$ of $\mathrm{FeCl}_{2}$ (3.0 mM), and $10 \mu \mathrm{L}$ of ferrozine $(5.0 \mathrm{mM})$ were mixed with $137 \mu \mathrm{L}$ of distilled water and incubated for $10 \mathrm{~min}$ at $25^{\circ} \mathrm{C}$. The Abs was then measured at $562 \mathrm{~nm}$.

$$
\mathrm{Fe}^{2+} \text { chelating rate }(\%)=\left(1-\frac{\mathrm{abs}_{1}-\mathrm{abs}_{2}}{\mathrm{abs}_{0}}\right) \times 100
$$

where $a b s_{0}$ is the abs of diluted water (instead of the sample), $\mathrm{abs}_{1}$ is the abs of the sample, and $\mathrm{abs}_{2}$ is the abs of the identical conditions as those of $\mathrm{abs}_{1}$ after replacing $\mathrm{FeCl}_{2}$ with distilled water.
Detection of Reducing Power. The reducing power was measured according to past research with minor modifications. $^{51}$ In brief, $50 \mu \mathrm{L}$ of the sample, $50 \mu \mathrm{L}$ of $\mathrm{K}_{3} \mathrm{Fe}(\mathrm{CN})_{6}$ $(1 \%, \mathrm{w} / \mathrm{v})$, and $50 \mu \mathrm{L}$ of PBS $(0.2 \mathrm{M}, \mathrm{pH}=6.6)$ were mixed in a 96-well plate and incubated for $20 \mathrm{~min}$ at $50{ }^{\circ} \mathrm{C}$. Then, $50 \mu \mathrm{L}$ of ferric trichloride and $30 \mu \mathrm{L}$ of $\mathrm{FeCl}_{3}(0.1 \%$, w/v) were added for termination and coloration, and the Abs was measured at $700 \mathrm{~nm}$.

$$
\text { reduction power }=\mathrm{abs}_{1}-\mathrm{abs}_{2}
$$

where $a b s_{1}$ is the abs of the sample and $a b s_{2}$ is the abs of the identical conditions as those of $\mathrm{abs}_{1}$ after replacing $\mathrm{FeCl}_{3}$ with distilled water.

Absolute Quantity Sequencing of 16S rRNA Amplicons. Absolute quantification of $16 \mathrm{~S}$ rRNA and basic data quality control were performed with a commercial kit from Genesky Biotechnologies Inc. (Shanghai, China). Briefly, total genomic DNA was extracted using the PowerSoil DNA Isolation Kit (MoBio, Carlsbad). Bacterial genomic DNA was used as a template to amplify the V3-V4 hypervariable region of the $16 \mathrm{~S}$ rRNA gene with the forward primer $\left(5^{\prime}\right.$ CCTACGGGNGGCWGCAG-3') and the reverse primer ( $5^{\prime}$ GACTACHVGGGTATCTAATCC-3'). For absolute quantification, multiple spike-ins with conserved regions identical to the $16 \mathrm{~S}$ rDNA and variable regions replaced by random sequences with $\sim 40 \%$ gas chromatography (GC) content were artificially synthesized, and an appropriate mixture with known gradient copy numbers of spike-ins was added to the sample DNA pools. All OTUs were then annotated based on the Ribosomal Database Project (RDP). Rarefaction analysis, alpha diversities (including the Shannon, Simpson, Chao1, and ACE indexes), and beta diversities were analyzed by $\mathrm{R}$ Project (Vegan package, V3.3.1).

SCFA Detection by Gas Chromatography (GC). An Agilent 7820A GC system (Agilent Technologies, Santa Clara, CA) was equipped with a flame ionization detector (FID), and a DB-FFAP capillary column (Agilent, $30 \mathrm{~m} \times 0.25 \mathrm{~mm} \times 0.25$ $\mu \mathrm{m})$ was used for component separation. The operating temperature conditions of the GC system were as follows: initial column temperature was $80{ }^{\circ} \mathrm{C}$ (held for $1 \mathrm{~min}$ ), then increased from 80 to $160{ }^{\circ} \mathrm{C}$ at a rate of $3{ }^{\circ} \mathrm{C} / \mathrm{min}$, and finally increased from 160 to $230^{\circ} \mathrm{C}$, which was held for $1 \mathrm{~min}$. Other parameters were set as follows: injection port temperature, 300 ${ }^{\circ} \mathrm{C}$; FID temperature, $320{ }^{\circ} \mathrm{C}$; and injection volume, $1 \mu \mathrm{L}$. The flow rates of dry air, hydrogen, and nitrogen were 300,30 , and $20 \mathrm{~mL} / \mathrm{min}$, respectively. Each experiment was performed in triplicate.

Statistical Analysis. The experimental data are expressed as the mean \pm standard deviation (SD) of triplicate measurements. All data were analyzed with GraphPad Prism 7.0 software, and the significance level of multiple comparisons was calculated by one-way analysis of variance (ANOVA), followed by Tukey's multiple comparisons test. $P<0.05$ was regarded as a statistically significant difference.

\section{ASSOCIATED CONTENT}

\section{Supporting Information}

The Supporting Information is available free of charge at https://pubs.acs.org/doi/10.1021/acsomega.1c03485.

High-performance liquid chromatography of MV after digestion of saliva, simulated gastric, and intestinal juice; UPLC-MS response value of MV and MVMs after in 
vitro incubation; and rarefaction curves, Shannon curves, and PCoA analysis of each treatment group (PDF)

\section{AUTHOR INFORMATION}

\section{Corresponding Author}

Ying Lin - Guangdong Key Lab Fermentation \& Enzyme Engineering, School of Biological Engineering, South China University of Technology, Guangzhou 510006, P. R. China; Email: feylin@scut.edu.cn

\section{Authors}

Ruiming Xiao - Guangdong Key Lab Fermentation \& Enzyme Engineering, School of Biological Engineering, South China University of Technology, Guangzhou 510006, P. R. China; (1) orcid.org/0000-0002-0862-5940

Wanci Liao - Guangdong Key Lab Fermentation \& Enzyme Engineering, School of Biological Engineering, South China University of Technology, Guangzhou 510006, P. R. China

Guangjuan Luo - Guangdong Key Lab Fermentation \& Enzyme Engineering, School of Biological Engineering, South China University of Technology, Guangzhou 510006, P. R. China

Zhenning Qin - Guangdong Key Lab Fermentation \& Enzyme Engineering, School of Biological Engineering, South China University of Technology, Guangzhou 510006, P. R. China

Shuangyan Han - Guangdong Key Lab Fermentation \& Enzyme Engineering, School of Biological Engineering, South China University of Technology, Guangzhou 510006, P. R. China

Complete contact information is available at:

https://pubs.acs.org/10.1021/acsomega.1c03485

\section{Author Contributions}

R.X. (first author) carried out and designed most of the experiments, analyzed microbiome and metabolic data, and composed the manuscript. W.L. helped organized data and carried out GC analysis of the short-chain fatty acids of incubation samples. G.L. helped collect the feces samples and organized the ethical review document and instructed the first author to conducted UPLC-MS. Z.Q. helped plot and incubation. Y.L. and S.H. were supervisors to design the project and revised the manuscript for publication.

\section{Notes}

The authors declare no competing financial interest.

\section{ACKNOWLEDGMENTS}

All of the authors are thankful for the financial support of the National Key Research and Development Program of China (Grant No. 2017YFD0400300) and the Key-Area Research and Development Program of Guangdong Province (Grant No. 2018B020205002).

\section{ABBREVIATIONS USED}

MV, mogroside V; FOS, fructooligosaccharide; BNM, basic nutrition growth medium; SCFAs, short-chain fatty acids; MVMs, mogroside $\mathrm{V}$ metabolites

\section{REFERENCES}

(1) Xia, Y.; Riverohuguet, M.; Hughes, B.; Marshall, W. Isolation of the sweet components from Siraitia grosvenorii. Food Chem. 2008, $107,1022-1028$.
(2) Pawar, R. S.; Krynitsky, A. J.; Rader, J. I. Sweeteners from plants-with emphasis on Stevia rebaudiana (Bertoni) and Siraitia grosvenorii (Swingle). Anal. Bioanal. Chem. 2013, 405, 4397-407.

(3) Additives, E. P. oF.; Flavourings; Younes, M.; Aquilina, G.; Engel, K. H.; Fowler, P.; Frutos Fernandez, M. J.; Furst, P.; Gurtler, R.; Gundert-Remy, U.; Husoy, T.; Mennes, W.; Moldeus, P.; Oskarsson, A.; Shah, R.; Waalkens-Berendsen, I.; Wolfle, D.; Degen, G.; Herman, L.; Gott, D.; Leblanc, J. C.; Giarola, A.; Rincon, A. M.; Tard, A.; Castle, L. Safety of use of Monk fruit extract as a food additive in different food categories. EFSA J. 2019, 17, No. e05921.

(4) Liu, X.; Zhang, J.; Li, Y.; Sun, L.; Xiao, Y.; Gao, W.; Zhang, Z. Mogroside derivatives exert hypoglycemics effects by decreasing blood glucose level in HepG2 cells and alleviates insulin resistance in T2DM rats. J. Funct. Foods 2019, 63, No. 103566.

(5) Qi, X. Y.; Chen, W. J.; Zhang, L. Q.; Xie, B. J. Mogrosides extract from Siraitia grosvenori scavenges free radicals in vitro and lowers oxidative stress, serum glucose, and lipid levels in alloxan-induced diabetic mice. Nutr. Res. 2008, 28, 278-284.

(6) Takasaki, M.; Konoshima, T.; Murata, Y.; Sugiura, M.; Nishino, H.; Tokuda, H.; Matsumoto, K.; Kasai, R.; Yamasaki, K. Anticarcinogenic activity of natural sweeteners, cucurbitane glycosides, from Momordica grosvenori. Cancer Lett. 2003, 198, 37-42.

(7) Liu, C.; Dai, L.; Liu, Y.; Dou, D.; Sun, Y.; Ma, L. Pharmacological activities of mogrosides. Future Med. Chem. 2018, $10,845-850$.

(8) Peng, M.; Tabashsum, Z.; Anderson, M.; Truong, A.; Houser, A. K.; Padilla, J.; Akmel, A.; Bhatti, J.; Rahaman, S. O.; Biswas, D. Effectiveness of probiotics, prebiotics, and prebiotic-like components in common functional foods. Compr. Rev. Food Sci. Food Saf. 2020, 19, $1908-1933$

(9) Morales, D.; Shetty, S. A.; Lopez-Plaza, B.; Gomez-Candela, C.; Smidt, H.; Marin, F. R.; Soler-Rivas, C. Modulation of human intestinal microbiota in a clinical trial by consumption of a beta-Dglucan-enriched extract obtained from Lentinula edodes. Eur. J. Nutr. 2021, 60, 3249-3265.

(10) Xu, J.; Chen, H. B.; Li, S. L. Understanding the Molecular Mechanisms of the Interplay Between Herbal Medicines and Gut Microbiota. Med. Res. Rev. 2017, 37, 1140-1185.

(11) Zhou, G.; Peng, Y.; Zhao, L.; Wang, M.; Li, X. Biotransformation of Total Saponins in Siraitia Fructus by Human Intestinal Microbiota of Normal and Type 2 Diabetic Patients: Comprehensive Metabolite Identification and Metabolic Profile Elucidation Using LC-Q-TOF/MS. J. Agric. Food Chem. 2017, 65, $1518-1524$

(12) Unno, T.; Hisada, T.; Takahashi, S. Hesperetin Modifies the Composition of Fecal Microbiota and Increases Cecal Levels of ShortChain Fatty Acids in Rats. J. Agric. Food Chem. 2015, 63, 7952-7.

(13) Koh, A.; De Vadder, F.; Kovatcheva-Datchary, P.; Backhed, F. From Dietary Fiber to Host Physiology: Short-Chain Fatty Acids as Key Bacterial Metabolites. Cell 2016, 165, 1332-1345.

(14) Zhao, L.; Zhang, F.; Ding, X.; Wu, G.; Lam, Y. Y.; Wang, X.; Fu, H.; Xue, X.; Lu, C.; Ma, J.; Yu, L.; Xu, C.; Ren, Z.; Xu, Y.; Xu, S.; Shen, H.; Zhu, X.; Shi, Y.; Shen, Q.; Dong, W.; Liu, R.; Ling, Y.; Zeng, Y.; Wang, X.; Zhang, Q.; Wang, J.; Wang, L.; Wu, Y.; Zeng, B.; Wei, H.; Zhang, M.; Peng, Y.; Zhang, C. Gut bacteria selectively promoted by dietary fibers alleviate type 2 diabetes. Science 2018, 359, 11511156.

(15) Zhang, Y.; Wu, Z.; Liu, J.; Zheng, Z.; Li, Q.; Wang, H.; Chen, Z.; Wang, K. Identification of the core active structure of a Dendrobium officinale polysaccharide and its protective effect against dextran sulfate sodium-induced colitis via alleviating gut microbiota dysbiosis. Food Res. Int. 2020, 137, No. 109641.

(16) Koppel, N.; Maini Rekdal, V.; Balskus, E. P. Chemical transformation of xenobiotics by the human gut microbiota. Science 2017, 356, No. eaag2770.

(17) El Kaoutari, A.; Armougom, F.; Gordon, J. I.; Raoult, D.; Henrissat, B. The abundance and variety of carbohydrate-active enzymes in the human gut microbiota. Nat. Rev. Microbiol. 2013, 11, 497-504. 
(18) Schwalm, N. D., 3rd; Groisman, E. A. Navigating the Gut Buffet: Control of Polysaccharide Utilization in Bacteroides spp. Trends Microbiol. 2017, 25, 1005-1015.

(19) Ju, P.; Ding, W.; Chen, J.; Cheng, Y.; Yang, B.; Huang, L.; Zhou, Q.; Zhu, C.; Li, X.; Wang, M.; Chen, J. The protective effects of Mogroside $\mathrm{V}$ and its metabolite 11-oxo-mogrol of intestinal microbiota against MK801-induced neuronal damages. Psychopharmacology 2020, 237, 1011-1026.

(20) Shen, H.; Gao, X. J.; Li, T.; Jing, W. H.; Han, B. L.; Jia, Y. M.; $\mathrm{Hu}$, N.; Yan, Z. X.; Li, S. L.; Yan, R. Ginseng polysaccharides enhanced ginsenoside $\mathrm{Rb} 1$ and microbial metabolites exposure through enhancing intestinal absorption and affecting gut microbial metabolism. J. Ethnopharmacol. 2018, 216, 47-56.

(21) Liang, H.; Cheng, R.; Wang, J.; Xie, H.; Li, R.; Shimizu, K.; Zhang, C. Mogrol, an aglycone of mogrosides, attenuates ulcerative colitis by promoting AMPK activation. Phytomedicine 2021, 81, No. 153427.

(22) Dabek, M.; McCrae, S. I.; Stevens, V. J.; Duncan, S. H.; Louis, P. Distribution of beta-glucosidase and beta-glucuronidase activity and of beta-glucuronidase gene gus in human colonic bacteria. FEMS Microbiol. Ecol. 2008, 66, 487-495.

(23) Ban, Q.; Cheng, J.; Sun, X.; Jiang, Y.; Zhao, S.; Song, X.; Guo, M. Effects of a synbiotic yogurt using monk fruit extract as sweetener on glucose regulation and gut microbiota in rats with type 2 diabetes mellitus. J. Dairy Sci. 2020, 103, 2956-2968.

(24) He, Y.; Wu, W.; Wu, S.; Zheng, H. M.; Li, P.; Sheng, H. F.; Chen, M. X.; Chen, Z. H.; Ji, G. Y.; Zheng, Z. D.; Mujagond, P.; Chen, X. J.; Rong, Z. H.; Chen, P.; Lyu, L. Y.; Wang, X.; Xu, J. B.; Wu, C. B.; Yu, N.; Xu, Y. J.; Yin, J.; Raes, J.; Ma, W. J.; Zhou, H. W. Linking gut microbiota, metabolic syndrome and economic status based on a population-level analysis. Microbiome 2018, 6, No. 172.

(25) Guarino, M.; Altomare, A.; Emerenziani, S.; Di Rosa, C.; Ribolsi, M.; Balestrieri, P.; Iovino, P.; Rocchi, G.; Cicala, M. Mechanisms of Action of Prebiotics and Their Effects on GastroIntestinal Disorders in Adults. Nutrients 2020, 12, No. 1037.

(26) O’Toole, P. W.; Marchesi, J. R.; Hill, C. Next-generation probiotics: the spectrum from probiotics to live biotherapeutics. Nat. Microbiol. 2017, 2, No. 17057.

(27) Pellock, S. J.; Walton, W. G.; Biernat, K. A.; Torres-Rivera, D.; Creekmore, B. C.; Xu, Y.; Liu, J.; Tripathy, A.; Stewart, L. J.; Redinbo, M. R. Three structurally and functionally distinct beta-glucuronidases from the human gut microbe Bacteroides uniformis. J. Biol. Chem. 2018, 293, 18559-18573.

(28) Yoshida, N.; M T E, M. D.; Tomoya Yamashita, M. D.; Watanabe, H.; Hayashi, T.; Tabata, T.; Hoshi, N.; Hatano, N.; Ozawa, G.; Sasaki, N.; Mizoguchi, T.; Amin, H. Z.; Hirota, Y.; Ogawa, W.; Yamada, T.; Hirata, K.-i. Bacteroides vulgatus and Bacteroides dorei Reduce Gut Microbial Lipopolysaccharide Production and Inhibit Atherosclerosis. Circulation 2018, 138, 2486-2498.

(29) Munukka, E.; Rintala, A.; Toivonen, R.; Nylund, M.; Yang, B.; Takanen, A.; Hanninen, A.; Vuopio, J.; Huovinen, P.; Jalkanen, S.; Pekkala, S. Faecalibacterium prausnitzii treatment improves hepatic health and reduces adipose tissue inflammation in high-fat fed mice. ISME J 2017, 11, 1667-1679.

(30) Liang, D.; Li, N.; Dai, X.; Zhang, H.; Hu, H. Effects of different types of potato resistant starches on intestinal microbiota and shortchain fatty acids under in vitro fermentation. Int. J. Food Sci. Technol. 2021, 56, 2432-2442.

(31) Metzler-Zebeli, B. U.; Newman, M. A.; Ladinig, A.; Kandler, W.; Grull, D.; Zebeli, Q. Transglycosylated starch accelerated intestinal transit and enhanced bacterial fermentation in the large intestine using a pig model. Br. J. Nutr. 2019, 122, 1-13.

(32) Mou, J.; Li, Q.; Shi, W.; Qi, X.; Song, W.; Yang, J. Chain conformation, physicochemical properties of fucosylated chondroitin sulfate from sea cucumber Stichopus chloronotus and its in vitro fermentation by human gut microbiota. Carbohydr. Polym. 2020, 228, No. 115359.

(33) Scanlan, P. D.; Shanahan, F.; Marchesi, J. R. Cultureindependent analysis of desulfovibrios in the human distal colon of healthy, colorectal cancer and polypectomized individuals. FEMS Microbiol. Ecol. 2009, 69, 213-221.

(34) Shang, Q.; Jiang, H.; Cai, C.; Hao, J.; Li, G.; Yu, G. Gut microbiota fermentation of marine polysaccharides and its effects on intestinal ecology: An overview. Carbohydr. Polym. 2018, 179, 173185.

(35) Rogowski, A.; Briggs, J. A.; Mortimer, J. C.; Tryfona, T.; Terrapon, N.; Lowe, E. C.; Basle, A.; Morland, C.; Day, A. M.; Zheng, H.; Rogers, T. E.; Thompson, P.; Hawkins, A. R.; Yadav, M. P.; Henrissat, B.; Martens, E. C.; Dupree, P.; Gilbert, H. J.; Bolam, D. N. Glycan complexity dictates microbial resource allocation in the large intestine. Nat. Commun. 2015, 6, No. 7481.

(36) Macia, L.; Tan, J.; Vieira, A. T.; Leach, K.; Stanley, D.; Luong, S.; Maruya, M.; Ian McKenzie, C.; Hijikata, A.; Wong, C.; Binge, L.; Thorburn, A. N.; Chevalier, N.; Ang, C.; Marino, E.; Robert, R.; Offermanns, S.; Teixeira, M. M.; Moore, R. J.; Flavell, R. A.; Fagarasan, S.; Mackay, C. R. Metabolite-sensing receptors GPR43 and GPR109A facilitate dietary fibre-induced gut homeostasis through regulation of the inflammasome. Nat. Commun. 2015, 6, No. 6734.

(37) De Vadder, F.; Kovatcheva-Datchary, P.; Goncalves, D.; Vinera, J.; Zitoun, C.; Duchampt, A.; Backhed, F.; Mithieux, G. Microbiotagenerated metabolites promote metabolic benefits via gut-brain neural circuits. Cell 2014, 156, 84-96.

(38) Bedford, A.; Gong, J. Implications of butyrate and its derivatives for gut health and animal production. Anim. Nutr. 2018, 4, 151-159.

(39) Hosseini, E.; Grootaert, C.; Verstraete, W.; Van de Wiele, T. Propionate as a health-promoting microbial metabolite in the human gut. Nutr. Rev. 2011, 69, 245-258.

(40) McDonald, J. A. K.; Mullish, B. H.; Pechlivanis, A.; Liu, Z.; Brignardello, J.; Kao, D.; Holmes, E.; Li, J. V.; Clarke, T. B.; Thursz, M. R.; Marchesi, J. R. Inhibiting Growth of Clostridioides difficile by Restoring Valerate, Produced by the Intestinal Microbiota. Gastroenterology 2018, 155, 1495-1507.e15.

(41) Young, I. S.; Woodside, J. V. Antioxidants in health and disease. J. Clin. Pathol. 2001, 54, 176-186.

(42) Huang, G. D.; Zhong, X. F.; Deng, Z. Y.; Zeng, R. Proteomic analysis of ginsenoside $\mathrm{Re}$ attenuates hydrogen peroxide-induced oxidative stress in human umbilical vein endothelial cells. Food Funct. 2016, 7, 2451-2461.

(43) Chen, W. J.; Wang, J.; Qi, X. Y.; Xie, B. J. The antioxidant activities of natural sweeteners, mogrosides, from fruits of Siraitia grosvenori. Int. J. Food Sci. Nutr. 2007, 58, 548-556.

(44) Di Meo, S.; Venditti, P. Evolution of the Knowledge of Free Radicals and Other Oxidants. Oxid. Med. Cell Longevity 2020, 2020, $1-32$.

(45) Santos, S. C.; Fortes, G. A. C.; Camargo, L. T. F. M.; Camargo, A. J.; Ferri, P. H. Antioxidant effects of polyphenolic compounds and structure-activity relationship predicted by multivariate regression tree. LWT 2021, 137, No. 110366.

(46) Zheng, Y. Z.; Deng, G.; Liang, Q.; Chen, D. F.; Guo, R.; Lai, R. C. Antioxidant Activity of Quercetin and Its Glucosides from Propolis: A Theoretical Study. Sci. Rep. 2017, 7, No. 7543.

(47) Chen, G.; Xie, M.; Wan, P.; Chen, D.; Ye, H.; Chen, L.; Zeng, X.; Liu, Z. Digestion under saliva, simulated gastric and small intestinal conditions and fermentation in vitro by human intestinal microbiota of polysaccharides from Fuzhuan brick tea. Food Chem. 2018, 244, 331-339.

(48) Smith, F.; Pan, X.; Bellido, V.; Toole, G. A.; Gates, F. K.; Wickham, M. S.; Shewry, P. R.; Bakalis, S.; Padfield, P.; Mills, E. N. Digestibility of gluten proteins is reduced by baking and enhanced by starch digestion. Mol. Nutr. Food Res. 2015, 59, 2034-2043.

(49) Zhou, G.; Wang, M.; Li, Y.; Xu, R.; Li, X. Comprehensive analysis of 61 characteristic constituents from Siraitiae fructus using ultrahigh-pressure liquid chromatography with time-of-flight mass spectrometry. J. Pharm. Biomed. Anal. 2016, 125, 1-14.

(50) Yuan, Q.; Xie, Y.; Wang, W.; Yan, Y.; Ye, H.; Jabbar, S.; Zeng, $\mathrm{X}$. Extraction optimization, characterization and antioxidant activity in 
vitro of polysaccharides from mulberry (Morus alba L.) leaves. Carbohydr. Polym. 2015, 128, 52-62.

(51) Wang, Y. Y.; Wang, C. Y.; Wang, S. T.; Li, Y. Q.; Mo, H. Z.; He,

J. X. Physicochemical properties and antioxidant activities of tree peony (Paeonia suffruticosa Andr.) seed protein hydrolysates obtained with different proteases. Food Chem. 2021, 345, No. 128765. 